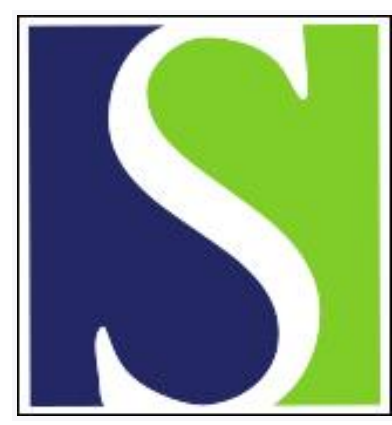

Scand J Work Environ Health 1994;20(5):313-321

https://doi.org/10.5271/sjweh.1391

Issue date: 01 Oct 1994

Cancer occurrence among workers exposed to acrylonitrile. by Rothman KJ

Affiliation: Epidemiology Resources, Inc., Boston, Massachusetts. 


\title{
Cancer occurrence among workers exposed to acrylonitrile
}

\author{
by Kenneth J Rothman, DrPH ${ }^{1}$
}

\begin{abstract}
ROTHMAN KJ. Cancer occurrence among workers exposed to acrylonitrile. Scand $J$ Work Environ Health 1994;20:313-21.

A MEDLINE search identified 12 published epidemiologic studies that have reported incidence or mortality experience among workers exposed to acrylonitrile. Many of the studies contain scanty descriptions of subject ascertainment, and most do not have good information on exposure assessment. Many also may have suffered from incomplete follow-up, as evinced by an overall deficit in the number of deaths observed, compared with the number expected from general population mortality rates. Such problems are not unique to studies on acrylonitrile, and to some extent they reflect the difficulties of conducting retrospective cohort studies. Despite these drawbacks, a simplified meta-analysis of the mortality experience reported for these cohorts revealed little evidence for carcinogenicity. Approximately the same number of cancer deaths was observed as was expected according to general population mortality rates (standardized mortality ratio $1.03,90 \%$ confidence interval $0.92-1.15$ ). The combined information from these studies is insufficient to support confidence about a lack of carcinogenicity at all sites. Nevertheless, despite the flaws in some of the individual studies, the summarized findings offer reassurance that workers exposed to acrylonitrile face no striking increases in mortality for all cancers or for respiratory cancer.
\end{abstract}

KEY TERMS - carcinogenesis, epidemiologic studies, lung cancer, occupational risk, respiratory cancer, review.

The purpose of this review is to assess the published epidemiologic evidence regarding the possible carcinogenicity of acrylonitrile, while focusing on respiratory cancer. To identify relevant publications, I searched MEDLINE for articles published during the past 15 years. At first the search key words were the title words acrylonitrile and cancer; I then searched using the title key words acrylonitrile and mortality, and subsequently using acrylonitrile as a subject instead of a title word, and occupational exposures, occupational illnesses, and adverse effects substituted for cancer and mortality. The search was then broadened even further to include articles with key words described as being related to acrylonitrile in volume 19 of the IARC Monographs on the Evaluation of the Carcinogenic Risk of Chemicals to Humans. These words included cyanoethylene, propenitrile, vinyl cyanide, acrylon, carbacryl, adiponitrile, nitrile elastomers, polyvinyl chloride, vinyl chloride monomer, butadiene, and styrene. Substituting butadiene or styrene for acrylonitrile provided a long list of studies, many of which were clearly not relevant to the topic of acrylonitrile, when judged by the title, abstract,

1 Epidemiology Resources, Inc, and Boston University School of Public Health, Boston, Massachusetts, United States.

Reprint requests to: Dr KJ Rothman, Epidemiology Resources Inc, One Newton Executive Park, Newton Lower Falls, MA 02162-1450, USA. and list of subject headings. After reviewing the abstracts of all the selected studies, I found 12 published papers that appeared to be epidemiologic studies of workers exposed to acrylonitrile with data on cancer morbidity or mortality $(1-12)$.

\section{Description and critique of the studies}

In this section I present a short summary, in chronological order, of each of 12 studies identified.

\section{Kiesselbach et al 1977.}

Kiesselbach et al carried out a retrospective cohort study (1) of 884 people working in the production and processing of acrylonitrile at the Bayer Leverkusen Division, which consists of 16 plants. The plants have produced acrylonitrile since 1929, but production fell during World War II and did not return to prewar levels until 1950. At that time, hygienic measures were poor, but they have improved steadily since about 1965 . The authors ascertained a cohort of 1973 male employees from worker lists; they then focused on a subset of employees with presumed exposure to acrylonitrile. With their having worked with acrylonitrile for at least $30 \%$ of their work time or having worked in a job with known high exposure to acrylonitrile as the criterion, 884 male employees with presumed exposure to acrylonitrile were identified. 
These 884 employees contributed a total of 13375 person-years of follow-up. Vital status was unknown for $6.8 \%$ of the cohort at the termination of the follow-up in 1977. The mortality experience of the workers was compared with the mortality rates of the population of Nordrhein-Westfalen during the period 1950-1977. The authors also conducted both a nested case-referent study that examined acrylonitrile exposure times for cancer cases and matched referents and a proportional mortality analysis.

The results indicated a total of 58 deaths, compared with 104.2 expected on the basis of NordrheinWestfalen rates. The deficit of observed to expected deaths is striking, and considerably greater than the deficit commonly seen in occupational mortality studies. Although the authors invoked the "healthy worker effect" (13) to explain the mortality deficit among cohort members, the deficit was greater than the $10-20 \%$ mortality advantage that is usually attributed to the healthy worker effect. Larger healthy worker effects are sometimes seen for workers with a short average tenure on the job (14), but another possible explanation for some or all of the deficit is that the ascertainment of deaths might have been incomplete.

The authors found 20 deaths due to "tumors" (presumably all or nearly all were cancers), compared with 20.4 expected. The close correspondence between the observed and expected rates should not necessarily be interpreted as indicating no excess, in view of the overall deficit of deaths in the cohort. It is possible that the overall deficit was attributable to an extremely strong healthy worker effect for noncancer causes of death, but it is also possible that there was an underascertainment of deaths that obscured a real excess of cancers in the cohort. Six deaths from respiratory cancer were observed, compared with 6.9 expected. Among the noncancer categories, there was a deficit of 15 deaths in the category of coronary heart disease (15 observed versus 30.4 expected) and a striking deficit of 10 deaths ( 2 observed versus 12.4 expected) for cerebrovascular disease. Accidents and other causes of death also showed large deficits. The proportional mortality analysis showed a proportional excess of cancer deaths with no excess of coronary deaths, a finding accentuating the concerns about completeness of follow-up.

Comment: This paper offers very little detail about some important topics. These topics include cohort ascertainment, exposure assessment, analytic methods, and certain specific categories of outcome, such as respiratory cancer. The reader is told that little information was available about the level of exposure. The authors fit a logistic model using data with varying lengths of follow-up. The logistic model, however, is not ideal for such data, and it is unclear how the authors handled the differing lengths of follow- up in these analyses. Other analyses took follow-up time into account more appropriately. The overall interpretability is hindered mainly by the lack of detail and the large deficit of deaths compared with the general population rates.

\section{Theiss et al 1980}

Theiss et al did a mortality study (2) of BASF workers exposed to acrylonitrile. BASF produces no acrylonitrile, but purchases it to use in the manufacture of styrene-acrylonitrile and acrylonitrile-butadienestyrene polymers, as well as other products. The study cohort comprised 1469 active and former workers from 12 BASF factories employed for at least six months in a job involving potential acrylonitrile exposure. No actual exposure assessment information was available. Follow-up was nearly complete $(98 \%)$ among the 1081 German workers, but the remainder of the cohort was foreign, and among these workers follow-up was poor (56\%). Mortality was compared with local, regional, and national mortality rates.

The cohort experienced 15350 person-years of follow-up, within which 89 deaths were observed. The expected number of deaths varied from 92 to 99 depending on whether the comparison was local, regional, or national. Of the 89 deaths, 27 were attributable to cancer, whereas only about 20.5 cancer deaths were expected. For lung cancer, 11 deaths were observed compared with 5.6 expected, accounting for most of the excess of cancer deaths. There were four deaths from lymphatopoietic cancers compared with 1.7 expected.

Comment: This paper also gives very little or no detail about (i) cohort ascertainment, including the eligibility dates for enrollment into the cohort; (ii) exposure assessment; and (iii) an examination of effects by duration or time of employment. In view of these shortcomings, very little can be inferred from this study.

\section{O'Berg 1980}

O'Berg's retrospective cohort study (3) comprised 1345 men who worked at the Du Pont Camden (South Carolina) plant anytime from its start-up in 1950 until the end of 1966 . Little detail was given about the ascertainment of the cohort. The cohort was followed through 1976. There was no quantitative exposure assessment available, but jobs were rated by a qualitative exposure assessment that included information from work history cards, interviews with supervisors, and, for salaried workers, a survey form.

Follow-up was based primarily on Du Pont's inhouse mortality information and cancer register, as well as a search of records of the Social Security Administration and the Department of Motor Vehicles in the state of South Carolina. The analysis com- 
pared the incidence and death rate from cancer and respiratory cancer of the workers with overall incidence and mortality data for all Du Pont employees. Although the analysis examined duration of exposure to acrylonitrile and job category, little detail was provided by category of outcome. A total of 89 deaths was observed, compared with an expected number of 77.4, based on Du Pont mortality rates, and 121.1, based on United States rates. A total of 25 cases of cancer was found, compared with 20.5 expected according to the Du Pont rates. There were eight cases of respiratory cancer, with 4.4 expected, and three cases of prostate cancer, with 0.9 expected.

Comment: Although this paper gives greater detail than the previous two, there was still an inadequate level of detail regarding most of the important areas of concern. For example, little is said about the cohort ascertainment and its completeness. There was, however, some indirect information on completeness. The author mentions that lung cancer occurred in two workers who should have been in the cohort but had not been included. Since there was a total of eight cases of lung cancer, this finding raises serious questions about the completeness of the cohort ascertainment. A separate validity check suggested that only $1 \%$ of the workers were missed in the cohort ascertainment, a figure that would be reassuring if not for the two cases of lung cancer among the purported $1 \%$ who were missed. The two additional cases of lung cancer were omitted from the analysis because their denominator experience was not included. If the ascertainment were in fact $99 \%$ complete, then the denominator should be within $1 \%$ of the correct denominator, and the cases should have been included in the analysis.

Another methodological problem comes from the comparison of the cohort's experience with the rates calculated from the Du Pont in-house registers. The registers record cancer occurrences and deaths among Du Pont workers who are actively employed or pensioned. This experience may differ from the morbidity and mortality experience of terminated and nonpensioned employees. The ascertainment of deaths in the study cohort was supplemented with a search of Social Security Administration records, in contrast to the Du Pont comparison rates. This methodological difference could explain the large difference in the expected numbers obtained with the Du Pont register data and the United States general population data.

\section{Werner \& Carter 1981.}

A retrospective mortality cohort study contributing 3774 person-years of follow-up for 1111 men who worked on the polymerization of acrylonitrile from 1950 through 1968 in six factories in the United Kingdom was carried out by Werner \& Carter (4). The follow-up extended through 1978. Essentially no information is given on the ascertainment of the cohort from the various factories. The intensity of exposure was assumed to be high for all of the men included in the study, but the time spent in the highexposure jobs was used to evaluate the effect of different durations of exposure. Comparison mortality rates were derived from the death rates for men in England and Wales.

Altogether 79 deaths were ascertained in the cohort, 68 of which occurred among those with at least one year of exposure. The expected number of deaths among those with at least one year of exposure was 72.4. For all cancers, there were 21 observed and 18.6 expected deaths in this subgroup. There were nine respiratory cancer deaths versus 7.6 expected, and five stomach cancer deaths versus 1.9 expected. The respiratory cancers included three deaths among workers aged 15-44 years, versus 0.7 expected. Deaths from accidents and suicides were more common than in the general population (13 observed versus 6.0 expected), but this excess pertained solely to the early years of the study. Cardiovascular deaths were fewer than expected.

The authors conducted a case-referent analysis of the duration of exposure using the three cases of lung cancer that occurred among workers aged 15-44 years. For referents, the authors chose a sample of all workers born in the same year as each of the three men with cancer and still alive at the time of the death of the cancer patients.

Comment: The paper provides no information on cohort ascertainment. The authors had little information on exposure assessment; the reader has only their assertion that exposure was heavy to aid in interpreting the results. Their analysis did not specify varying induction periods after exposure to look for a specific effect from the induction period. Instead they presented an analysis that confounds exposure duration with induction time. The strongest feature of this study was the relatively high overall standardized mortality ratio (SMR) $(68 / 72.4=0.94)$, which can be taken as an indicator of relatively thorough follow-up. There was little information available to the authors by which they might have evaluated or controlled for confounding. The case-referent analysis of the three young cases of lung cancer was not very informative.

\section{Delzell \& Monson 1982}

Delzell \& Monson performed a retrospective cohort study (5) of mortality among 327 employees who had "potential exposure" to acrylonitrile in a rubber chemicals plant. The subjects worked at a plant in Akron, Ohio, for at least two years in the interval from 1 January 1940 to 1 July 1971 . The number of person-years of follow-up that these workers contributed to the analysis was not reported. Mortality experience was assessed from the beginning of 1940 
or from the date of first employment in a relevant job until 1 July 1978. The comparison group was all white men in the United States. No exposure assessment information was given. Follow-up information was obtained from the company and from the Social Security Administration. All those not identified as dead were assumed to be alive.

Overall, there were 74 deaths compared with 89.5 expected, 22 cancer deaths compared with 17.9 expected, and 9 lung cancer deaths compared with 5.9 expected. The lung cancer deaths appeared to be related to a longer duration of employment.

Comment: This study began follow-up for each worker at the start of employment, but workers were ineligible for the cohort unless they had worked for two years in one of two relevant departments. Those two years were "immortal person-years"; every study subject had to have survived those two years to be eligible for the study. Consequently, the first two years of follow-up for each worker should have been excluded from the person-time calculations. This problem could not have led to a serious underestimation of the mortality of the workers in the study, however, since the amount of immortal person-time was small relative to the overall person-time. The authors also examined induction time starting from time of first employment, as opposed to starting from the time at which a stated amount of job exposure had occurred, and thus duration of employment was confounded by induction time. The follow-up seems to be reasonably complete when judged by the relatively high SMR of 0.83 (74/89.5), although this judgment presumes that there was no great excess expected for all deaths.

\section{O'Berg et al 1985}

O'Berg and her colleagues (6) extended the followup experience of the cohort reported by O'Berg in 1980 (3). Surveillance for cancer incidence was extended through 1983 and mortality surveillance through 1981. The information in this report apparently contains all the events already reported so that it supersedes the previous one. The authors observed a total of 155 deaths, compared with 134.5 expected according to company-wide data. The excess of approximately $15 \%$ over expected for all deaths was also found for cancer deaths, of which 36 were observed versus 31.6 expected. Excluding salaried workers, there were 31 cancer deaths with 27.0 expected. Cancer mortality did not vary much from the expected numbers of deaths by site of occurrence. There were 12 lung cancer deaths observed versus 10.2 expected for wage workers and 14 observed versus 11.6 expected for wage and salaried workers combined.

The cancer incidence findings were not very different from the cancer mortality findings. There was a total of 43 incident cases of cancer observed ver- sus 36.7 expected according to company-wide rates. For lung cancer there were 10 cases observed versus 7.2 expected. There are fewer incident lung cancer cases than deaths because of the differing time periods for follow-up. (The incidence data refer to the period 1956-1983, whereas the mortality data refer to 1950-1981.) For prostate cancer, there were six cases observed and 1.8 expected. All six cases of prostate cancer occurred among wage workers, for whom the expected number was 1.5. There were seven cases of lymphopoietic cancer versus 3.7 expected, and for the wage workers the respective numbers were 6 versus 2.9 expected. The only analysis relating the amount of cumulative exposure or induction time to any effect was for lung cancer; this analysis showed seven cases of lung cancer versus 3.5 expected among workers with more than 20 years since first exposure (the category of longest duration), and six cases versus 2.8 expected for workers with the highest category of cumulative exposure. Though the numbers were small, it is nevertheless worth noting that the greatest ratio of observed to expected was for those in the combined category of long induction period and greatest cumulative exposure, in which there were four cases versus $1.8 \mathrm{ex}-$ pected.

Comment: Since these data presumably comprise the earlier findings of O'Berg (3), the report should be viewed as a replacement for, rather than as andition to, the earlier paper. The relatively high overall SMR may reflect incomplete death ascertainment in the Du Pont mortality register used to generate the expected numbers. The primary difference between these and the earlier data is the finding on prostate cancer. The lung cancer excess, while modest overall, appears to be related to the amount of exposure.

\section{Chen et al 1987}

Another report on Du Pont workers, but from a textile fibers plant that manufactured orlon in Waynesboro, Virginia, was published by Chen et al (7). Acrylonitrile is a main ingredient in orlon. Production began in 1957, but exposure began in 1944 with research activities at the plant. From plant records of work histories, the authors identified 1083 male workers with potential exposure to acrylonitrile between 1944 and 1970 at the plant. A committee was established to review exposure data and to classify the exposure levels (low, moderate, or high) for specific jobs. Mortality follow-up for active and pensioned employees was ascertained through the Du Pont mortality register, which began in 1957. Additional follow-up was through a search of records kept by the Social Security Administration. Followup extended through 1981.

There were 92 deaths observed in the cohort during the follow-up period, substantially fewer than the 124.0 expected on the basis of Du Pont mortality 
rates and the 177.2 expected on the basis of rates for all white males in the United States. The deficit in deaths was more striking for salaried employees than for wage employees. Among the wage workers, there were 18 cancer deaths versus 20.4 expected from the Du Pont rates and 24.1 expected from the United States rates. There were seven deaths from lung cancer among the wage workers, compared with 7.9 expected on the basis of the Du Pont rates. The authors reported no trend by duration of employment or by time period of follow-up.

As in the study by O'Berg et al (6), the authors examined cancer incidence in the cohort. There were 37 cases of cancer identified during the period 1956-1983. There was no excess seen for lung cancer ( 5 cases observed versus 6.9 expected), but there was an excess for prostate cancer ( 5 observed versus 1.9 expected). The prostate cases were concentrated in the most recent years of follow-up.

Comment: This study reports little detail about cohort ascertainment or data analysis. It may be that the small size of the study inhibited more detailed reporting. The authors reported a lack of "statistically significant" trends in their mortality analyses, but they did not show the analyses. Given the somewhat scanty data, it may be that some substantial trends were present but were not "statistically significant." The difference in expected numbers in the comparison with Du Pont company-wide rates and the United States comparison rates, as in the earlier Du Pont studies, is notable.

\section{Chen et al 1988 a}

The paper by Chen et al (8), relating only to cancer incidence, is another retrospective cohort study on Du Pont workers. Most workers exposed to acrylonitrile are also exposed to dimethylformamide. The paper did not make clear exactly which population was studied, but it appears as if this is the same cohort as that reported by O'Berg in 1980. Most of the findings relate to dimethylformamide. Only 16 employees had exposure to acrylonitrile with no exposure to dimethylformamide; therefore no analysis was reported that pertained to acrylonitrile alone.

Comment: As the findings of this study relate primarily to dimethylformamide, it does not appear to be as contributory as the other studies regarding effects of acrylonitrile. Exposure to dimethylformamide predominated over exposure to acrylonitrile, and the analysis reflected this imbalance of exposure.

\section{Chen et al $1988 b$}

Chen and her co-workers' carried out another study (9) that was a companion paper to the previous one (8). It reports the findings for mortality. As for the previous paper, the findings relate primarily to dimethylformamide rather than to acrylonitrile exposure.

\section{Collins et al 1989}

A retrospective cohort study by Collins et al (10) reports the effects of acrylonitrile exposure on workers at two plants of the American Cyanamid Company. The two cohorts comprised 2671 men who worked during the period $1951-1973$ at one plant and 1957-1973 at the other plant. Follow-up for mortality continued through 1983 with the use of the Social Security Administration and the National Death Index to find deaths. Exposure assessment included industrial hygiene measurements in 1977, which the authors indicated was representative of exposures going back to the beginning of plant operations. Insofar as they could, the authors used jobspecific exposure measurements, with some adjustments for changes in handling practices and hygienic improvements with time. Unlike the other studies reviewed, this one had smoking information available, although only for $58 \%$ of the workers. The mortality of the cohorts was compared with "general population mortality rates," but the authors did not state what the general population was. The authors also compared the mortality of workers who had a defined level of exposure with that of unexposed workers.

The total number of deaths among exposed workers was 145, for an overall SMR of 0.67 . The relatively large number of deaths made this the largest study of acrylonitrile exposure. Despite the sizeable deficit relative to the expected number for total deaths, the number of cancer deaths $(\mathrm{N}=43)$ was about equal to the expected number (SMR 1.01). The 15 lung cancer deaths observed were also about the same as the number expected according to the general population comparison. There were two deaths from prostate cancer compared with 1.49 expected. Internal comparisons by level of intensity of exposure to acrylonitrile showed a modest $U$-shaped curve in the effect estimates for lung cancer mortality, with very little overall linear trend; the relative risk estimates reported for the four exposure categories were 1.1 for exposure under $0.01 \mathrm{ppm}$ per year, 0.7 for exposure in the range of $0.01-0.7 \mathrm{ppm}$ per year, 0.7 for exposure of $0.7-7.0 \mathrm{ppm}$ per year, and 1.2 for exposure greater than $7.0 \mathrm{ppm}$ per year. No internal comparison was presented outside of the analysis for lung cancer.

Comment: This paper puts too much emphasis on statistical significance, and it is difficult to determine whether some potentially revealing results were simply not presented because the authors did not find them to be statistically significant. Very little information is given about the ascertainment of the study subjects, and very little detail is given in the analysis. The exposure information is better than that in 
many of the other studies, being based on job-specific measurements.

\section{Zhou \& Wang 1991}

Zhou \& Wang's paper (11) is a report of a retrospective cohort study of 1811 workers in a Chinese chemical fiber factory in Fushun, China. The workers were exposed to acrylonitrile, methyl acrylate, and thiocyanate. Acrylonitrile exposure was reported to have ranged from a low of $0.057 \mathrm{mg} \cdot \mathrm{m}^{-3}$ to a high of $4.12 \mathrm{mg} \cdot \mathrm{m}^{-3}$, the average being 1.24 (SD 1.16) $\mathrm{mg} \cdot \mathrm{m}^{-3}$. The cohort was ascertained from factory records and included all workers at the factory, beginning in 1971, when production began, and continuing through 1988. The paper stated that work records were used to ascertain deaths. The investigators claim to have mailed surveys to workers who moved or retired. There were 115 individuals out of the 1811 who could not be located; they were dropped from the study.

Forty-two deaths were observed among the members of the cohort. Death rates for employees were compared with the rates for residents of Fushun. The authors also claimed to have compared the worker experience with rates for all of China, but I could not find these comparisons presented in the paper. The analysis shows an excess in rates for death from all causes combined (27\%) and for all tumors $(25 \%)$. Among the 13 who died from diseases of the circulatory system, 10 had cerebrovascular disease, compared with about 3.8 expected cases. For the other three circulatory deaths, the expected number was about 5.8, as calculated from numbers based on data provided in the tables. The authors presented an analysis examining death rates for all causes, cancer, and cerebrovascular disease according to the number of years worked. These analyses showed markedly increasing trends for all three sets of rates with the number of years worked. They also included an anal$y$ sis of age of death for various categories of death.

Comment: There are too many unaddressed questions about the methodology of this study to draw any reasonable inferences from it. The paper gives scant information on how the exposure measurements were obtained, or to whom they refer. There is no mention of any attempt to assess exposure individually for workers assigned different jobs. The authors attempted to include all workers at the plant. Some workers had moved or retired, and these former employees were surveyed by mail. No reason was given for the survey being sent, nor was any indication given of what information was obtained. The survey might have been an attempt at follow-up for vital status on these former employees, but the authors claim that the 115 nonrespondents to the survey were dropped from the study. Since dead individuals would not be responding, the survey could hardly have been an attempt to ascertain vital status.
The death information for the workers may not be comparable with the death information for the general population, inasmuch as the authors stated that the death information for workers came from factory records, whereas death information for the general population presumably did not. The methods for follow-up were not described. The analysis is sketchy and difficult to interpret. The number of cerebrovascular deaths appeared to be large, but more so in comparison with the experience of western populations than in comparison with Asian populations. Despite the fact that about three-quarters of the vascular deaths had cerebrovascular causes, a proportion unusually high for any western population, the SMR (on comparison with the Fushun population) was less than 3 . While this is still an excess, its magnitude is not as striking as one might infer from the high proportion of vascular deaths that were cerebrovascular.

The strong trends with years of employment for deaths from all causes and cancer deaths appear to have been based on crude rates, uncontrolled for potential confounding by age, which could have been substantial. Age is generally strongly correlated with number of years worked, since both increase with time. The sharply increasing trend in SMR with duration of work for all causes of death indicates that confounding by age has not been controlled. It is implausible that workplace exposures could cause a duration-related effect with such a striking increase in deaths from all causes. On the other hand, age is linearly related to duration of work and could have accounted for a strong increase in the rate of death from all causes. The authors also present age-at-death analyses, which are always invalid because they ignore the age structure of the compared groups. The correct approach would be to compare age-specific death rates, which is basically what the calculation of SMR values is intended to accomplish.

Overall, weaknesses in the study design and analysis are severe enough to preclude any meaningful inferences from this paper. Not much can be inferred about the notable excess of cerebrovascular deaths, given the study's methodological weaknesses.

\section{Swaen et al 1992}

Swaen et al carried out a retrospective cohort study (12) of 2842 Dutch workers with potential exposure to acrylonitrile on the job. The workers were employed at one of eight chemical companies and worked in a job with potential acrylonitrile exposure for at least six months during the interval from 1 January 1956 through 1 July 1979 . A task force of investigators reviewed personnel files for the participating companies and assembled information on work histories. Only Dutch workers living in The Netherlands were included, to ensure comparability with the national mortality data. The study also included the follow-up of an unexposed cohort of 
3961 workers, assembled from the same companies with similar eligibility criteria and followed with the same methods.

The paper provides reasonably good detail on the exposure assessment, which of necessity was a hybrid methodology consisting of measurements from some companies and indirect assessments based on interviews with key personnel. Jobs were classified into exposure level ranges. Use of respiratory protection was documented for various jobs, but it was not taken into account when the exposure scores were assigned. Therefore actual exposure was probably lower than the assessed exposure in many cases, particularly workers with high levels of peak exposure, for which respiratory protection was mandatory. Both average and peak exposure levels were taken into account when the jobs were classified. The investigators assigned annual exposure scores for each job group, and these scores were reviewed by industrial hygienists from the individual companies. Cumulative doses were calculated for each worker according to the job exposure assessments and the time spent in specific jobs. Follow-up was evaluated with the use of the municipal population registers in The Netherlands and the death records kept by the Central Bureau of Statistics in The Netherlands.

Overall, in the exposed cohort, there were 134 deaths observed, with 172.7 expected according to the national mortality rates. Approximately the same ratio of observed to expected deaths was found for the unexposed cohort as for the exposed cohort. There were 42 cancer deaths in the observed cohort, with an expected number of 50.8, giving a SMR of 0.83 , not very different from that for all deaths. For lung cancer, there were 16 deaths in the exposed cohort compared with 19.5 expected, which gives a SMR for lung cancer of 0.82 . When lung cancer risk was examined according to three categories of dose, there was a marginal excess ( 8 deaths versus 7.2 expected) in the highest dose category and deficits for the smaller dose categories. The authors attributed the rising SMR by dose category to a waning of the healthy worker effect for longer-term employees. This interpretation was supported by the trend in the ratio of all deaths to expected deaths by dose category: for low dose ( $<1 \mathrm{ppm}$-years), the ratio was 0.67 ; for moderate dose ( $1-10 \mathrm{ppm}$-years) it was 0.78 ; and for high dose ( $\geq 10 \mathrm{ppm}$-years) it was 0.83 .

There were two prostate cancer deaths in the exposed cohort, compared with 0.5 expected. These two deaths occurred in the group with the highest exposure and the longest "latency."

Comment: This study is an important one from several points of view. It is by some criteria even larger than the study by Collins et al. Exposure assessment was a strong feature of this work in that it permitted a meaningful look at the experience of more heavily exposed workers. The exposure assessment was not able to take into account the use of protective gear, but otherwise it employed available information about level of exposure to the fullest. The follow-up was based on a good system of death registration, and the overall SMR of 0.78 for all deaths is a value typical of occupational cohorts with relatively complete follow-up of cohorts with no severe elevation in risk. For statistical reasons, the authors chose to avoid comparing the exposed cohort directly with the unexposed cohort, which would have been a stronger comparison than comparing both groups with outside data. Their overall findings are mostly reassuring about the lack of an effect of acrylonitrile exposure on lung cancer risk. On the other hand, the excess for prostate cancer, small in absolute terms but larger in relative terms, corroborates the findings of several other reports.

\section{Overall assessment}

The variety of results and the variety of concerns about validity in the reviewed studies pose a challenge in attempting to come to a unified interpretation. Most of the studies were small, and most were subject to criticisms about exposure assessment, methods or completeness of follow-up, and analytic techniques. Such problems are not unique to studies on acrylonitrile, and to a large extent they reflect the difficulties of conducting retrospective occupational cohort studies.

One approach to overcoming the problem of small numbers in these studies is to derive a quantitative assimilation of results - a "meta-analysis." While a meta-analysis does overcome the problem of statistical instability from numerous small studies, it has some drawbacks of its own. First, it combines studies that have differing degrees of validity. The hope in a meta-analysis is that unquantifiable biases that vary in direction and magnitude will cancel one another. A second problem is that meta-analyses are premised on the assumption that without biases, all the studies examined should give the same result. Biological conditions vary, however, and it may be that real heterogeneity is responsible for a spectrum of different results among the different studies. Averaging studies across such heterogeneous situations may only obscure the underlying pattern. Knowledge of the heterogeneity may be even more important than obtaining a single summary result. Third, any attempt to combine various studies faces the obstacle that the types of exposures and outcomes reported differ to varying degrees; thus any combination of studies will inevitably blur some possibly important distinctions in the definition of the study problem.

These problems should be borne in mind when any quantitative summary of these studies on acrylonitrile are considered. Table 1 gives just such a summary, based on the results from most of the studies included in this review. Inasmuch as the 
Table 1. Summary of observed and expected numbers of cancer and respiratory cancer deaths in published epidemiologic studies on acrylonitrile. ${ }^{\mathrm{a}}$ (SMR $=$ standardized mortality ratio)

\begin{tabular}{|c|c|c|c|c|}
\hline \multirow[b]{2}{*}{ Study } & \multicolumn{2}{|c|}{ All cancer } & \multicolumn{2}{|c|}{ Respiratory cancer } \\
\hline & $\begin{array}{c}\text { Observed } \\
\text { deaths }\end{array}$ & $\begin{array}{l}\text { Expected } \\
\text { deaths }\end{array}$ & $\begin{array}{c}\text { Observed } \\
\text { deaths }\end{array}$ & $\begin{array}{c}\text { Expected } \\
\text { deaths }\end{array}$ \\
\hline $\begin{array}{l}\text { Kiesselbach et al, } 1979(1) \\
\text { Thiess et al, } 1980 \text { (2) } \\
\text { Werner \& Carter, } 1981(4) \\
\text { Delzell \& Monson, } 1982(5) \\
\text { O'Berg et al, } 1985(6) \\
\text { Chen et al, } 1987(7) \\
\text { Collins et al, } 1989(10) \\
\text { Swaen et al, } 1992(12)\end{array}$ & $\begin{array}{l}20 \\
27 \\
21 \\
22 \\
31 \\
18 \\
43 \\
42\end{array}$ & $\begin{array}{l}20.4 \\
20.5 \\
18.6 \\
17.9 \\
27.0 \\
20.4 \\
42.6 \\
50.8\end{array}$ & $\begin{array}{r}6 \\
11 \\
9 \\
9 \\
12 \\
7 \\
15 \\
16\end{array}$ & $\begin{array}{r}6.9 \\
5.7 \\
7.6 \\
5.9 \\
10.2 \\
7.9 \\
15.7 \\
19.5\end{array}$ \\
\hline Total & 224 & 218.2 & 85 & 79.4 \\
\hline $\begin{array}{l}\text { Summary SMR (observed/expected) } \\
90 \% \text { confidence interval }\end{array}$ & \multicolumn{2}{|c|}{$\begin{array}{c}1.03 \\
0.92-1.15\end{array}$} & \multicolumn{2}{|c|}{$\begin{array}{l}1.07 \\
0.89-1.28\end{array}$} \\
\hline
\end{tabular}

a When available, data for wage earners, rather than data for all workers, are given. Under respiratory deaths, the figures refer to all respiratory deaths unless this information was not available, in which case the data refer to lung cancer deaths. The first study by O'Berg has been omitted because the same data are included in the later O'Berg study. Data from the study by Werner \& Carter are limited to those who had at least one year of exposure. The two papers by Chen et al in 1988 have been omitted because they report on exposure mainly to dimethylformamide, and one reports only incidence. The paper by Zhou \& Wang has been omitted because the validity of the data is questionable.

main concern about acrylonitrile relates to a possible carcinogenic effect, particularly respiratory cancer, the table focuses on the results for all cancer and for respiratory system cancer. To keep the results comparable and to avoid some duplication of data, those few reports that deal with cancer incidence have been omitted - the table includes only mortality results. When findings from a study were available for wage workers separately, as opposed to salary and wage workers combined, the data for wage workers only was included. The reason for this restriction is that the exposure was probably more intense among wage workers, and, if any effect of exposure occurred, it would presumably be stronger among wage workers.

The simplest type of meta-analysis is possible for SMR values. The observed and expected numbers of events that are the components of an SMR are already inherently weighted for precision, since the magnitude of each reflects the amount of person-time experienced by the cohort. By adding together the observed and expected numbers, respectively, from all studies, one obtains a summary measure that is weighted for study size. In addition, the summary measure that results from comparing the observed number summed over studies and the expected number summed over studies remains internally standardized, to the extent that the same confounders are controlled in each of the studies. Thus, if the SMR for each of the studies has been standardized for age, the summary SMR over all studies will also be standardized for age. Additional analyses, not shown, that combined these studies after weighting them by a subjective validity score, or that considered in addition those studies not listed in table 1 , produced results very close to the summary SMR values in table 1 .
As the table shows, the weighted total for the observed number of deaths is close to the total for the expected number of deaths for all cancers (SMR 1.03 ) and for respiratory cancers (SMR 1.07). These findings are a quantitative indication that in the aggregate these studies do not show a strong relation between work in an environment in which there is the possibility of exposure to acrylonitrile and subsequent death from cancer, respiratory cancer in particular.

The $90 \%$ confidence interval (15) for the SMR for all cancer deaths is $0.92-1.15$, and the $90 \%$ confidence interval for lung cancer is $0.89-1.28$. The upper limits of these intervals indicate that the combined data are not statistically compatible with very large increases in the risk of cancer death. On the other hand, because they attempt to summarize the precision of results from several different studies, these confidence intervals have less meaning than usual. The actual variability of the summary result is undoubtedly subject to more sources of error than the simple Poisson variation that the calculation presumes (16). Thus the relatively narrow confidence intervals should not be interpreted too literally as indicating that the summary result is a highly precise measure of little excess risk of death.

The following caveats should also be considered in this summary interpretation:

1. The number of observed deaths from all causes for the studies in table 1 was 815 , compared with an overall expected number of 1006.0, for an overall SMR of 0.81 . The roughly equal number of observed and expected cancer deaths does not necessarily indicate an absence of a relation, given the deficit of noncancer deaths that occurred in relation to the general population rates (17). 
2. Although O'Berg et al (6) reported larger relative excesses among workers with greater amounts of cumulative exposure, this finding was not replicated. An argument could be made that the null results of the study of Swaen et al contained a trend in the same direction. Swaen et al attributed this small trend to a diminishing healthy worker effect with increasing duration of employment, a reasonable hypothesis in view of the fact that no large excess was found even in the high-exposure category. The other studies generally did not have or present enough detailed data with which to evaluate high levels of exposure. Because of the lack of consistent information, findings on trend with exposure intensity or cumulative dose were not taken into account in the table.

3. Even aggregated over studies, these data are sparse with respect to many sites of cancer. It may well be that acrylonitrile is carcinogenic at some sites, but there is insufficient information in these studies to see it. For example, excesses for prostate cancer and stomach cancer were reported in a few studies. The excess of prostate cancer in the Du Pont studies was ascribed to nonoccupational factors by Walrath et al (18). Unfortunately, although it would be interesting to summarize the number of observed and expected cancer deaths for some sites other than respiratory cancer, there was not enough consistency of reporting for any other site to enable such a summary. One concern is that, when a study does not report on a given site of cancer, it is because there is no excess for that site. Some of the studies may have data that would balance the individual reports of positive findings for specific sites, but simply fail to show it.

4. Exposure assessment was weak for many of the studies, and there may have been considerable nondifferential misclassification of acrylonitrile exposure. Among workers heavily exposed to acrylonitrile, there may be a substantial effect of exposure on cancer risk that is attenuated in analyses that mix these workers with others who sustained smaller exposures.

These concerns notwithstanding, the combined data from the reviewed studies do offer reassurance that workers exposed to acrylonitrile apparently have experienced no striking excesses of mortality for all cancers or for respiratory cancer.

\section{Acknowledgments}

This work was supported by contracts with the Acrylonitrile Group and with the Monsanto Corporation.

\section{References}

1. Kiesselbach N, Korallus U, Lange HJ, Neiss HJ, Zwingers T. Bericht über eine prospektive epdemiologische studie mit zurückverlegtem eginn bei mitarbeitern des Leverkusener Werkes der Bayer AG mit acrylnitril (ACN) exposition [A report on a prospective epidemiologic study of workers at the Leverkusen plant of Bayer AG with acrylonitrile (ACN) exposure]. Zentralbl Arbeitsmed Abeitsschutz Prophyl Ergon 1979;29(10):256-9.

2. Thiess AM, Frentzel-Beyme R, Link R, Wild H. Mortalitätsstudie bei chemiefacharbeitern verschiedener produktionsbetriebe mit exposition auch gegenüber acrylnitril [Mortality study in chemical personnel of various industries exposed to acrylonitrile]. Zentralbl Arbeitsmed Arbeitsschutz Prophyl Ergon 1980;30(7): 259-67.

3. O'Berg MT. Epidemiologic study of workers exposed to acrylonitrile. J Occup Med 1980;22(4):245-52.

4. Werner JB, Carter JT. Mortality of United Kingdom acrylonitrile polymerisation workers. $\mathrm{Br} \mathbf{J}$ Ind Med 1981;38(3):247-53.

5. Delzell E, Monson RR. Mortality among rubber workers exposed to acrylonitrile. J Occup Med 1982; 24(10):767-9.

6. O'Berg, Chen JL, Burke CA, Walrath J, Pell S. Epidemiologic study of workers exposed to acrylonitrile: an update. J Occup Med 1985;27(11):835-40.

7. Chen JL, Walrath J, O'Berg MT, Burke CA, Pell S. Cancer incidence and mortality among workers exposed to acrylonitrile. Am J Ind Med 1987;11(2): $157-63$.

8. Chen JL, Fayerweather WE, Pell S. Cancer incidence of workers exposed to dimethylformamide and/or acrylonitrile. J Occup Med 1988;30:813-18.

9. Chen JL, Fayerweather WE, Pell S. Mortality study of workers exposed to dimethylformamide and/or acrylonitrile. J Occup Med 1988;30(10):819-21.

10. Collins JJ, Page LC, Caporossi JC, Utidjian HM, Saipher J. Mortality patterns among employees exposed to acrylonitrile. J Occup Med 1989;31(4):368 71.

11. Zhou B, Wang T. Historical cohort study of causes of death in a chemical fiber factory [in Chinese]. J Chinese Med Univ 1991;20:35-7.

12. Swaen GMH, Bloemen LJN, Twisk J, Scheffers T, Slangen JJM, Sturmans F. Mortality of workers exposed to acrylonitrile. J Occup Med 1992;34:801—9.

13. Checkoway H, Pearce NE, Crawford-Brown DJ. Research methods in occupational epidemiology. New York, NY: Oxford University Press, 1989:78-9.

14. Wen CP, Tsai SP, Gibson RL. Anatomy of the healthy worker effect: a critical review. J Occup Med 1983; 25:283-9.

15. Rothman KJ, Boice JD Jr. Epidemiologic analysis with a programmable calculator. Boston, MA: Epidemiology Resources Inc, 1982.

16. Greenland S. Randomization, statistics and causal inference. Epidemiology 1990;1:421—9.

17. Park RM, Maizlish NA, Punnett L, Moure-Eraso R, Silverstein M. A comparison of PMRs and SMRs as estimators of occupational mortality. Epidemiology 1991;2:49-59.

18. Walrath J, Fayerweather WE, Gilby PG, Pell S. A case-control study of cancer among Du Pont employees with potential for exposure to dimethylformamide. J Occup Med 1989;31:432-8.

Received for publication: 18 February 1994 